\title{
Comparison of numerical solution strategies for gravity field recovery from GOCE SGG observations implemented on a parallel platform
}

\author{
R. Pail and G. Plank \\ Institute of Geodesy, Department of Theoretical Geodesy, Graz University of Technology, Steyrergasse 30, A-8010 Graz, \\ Austria
}

\begin{abstract}
The recovery of a full set of gravity field parameters from satellite gravity gradiometry (SGG) is a huge numerical and computational task. In practice, parallel computing has to be applied to estimate the more than 90000 harmonic coefficients parameterizing the Earth's gravity field up to a maximum spherical harmonic degree of 300. Three independent solution strategies, i.e. two iterative methods (preconditioned conjugate gradient method, semi-analytic approach) and a strict solver (Distributed Non-approximative Adjustment), which are operational on a parallel platform ('Graz Beowulf Cluster'), are assessed and compared both theoretically and on the basis of a realistic-as-possible numerical simulation, regarding the accuracy of the results, as well as the computational effort. Special concern is given to the correct treatment of the coloured noise characteristics of the gradiometer. The numerical simulations show that there are no significant discrepancies among the solutions of the three methods. The newly proposed Distributed Nonapproximative Adjustment approach, which is the only one of the three methods that solves the inverse problem in a strict sense, also turns out to be a feasible method for practical applications.
\end{abstract}

Key words. Spherical harmonics - satellite gravity gradiometry - GOCE - parallel computing - Beowulf cluster

\section{Introduction}

The Earth's gravitational field is the focus of attention of the already launched or currently planned dedicated gravity field satellite missions: CHAMP (CHAllanging Minisatellite Payload; Reigber et al., 1999), GRACE (Gravity Recovery And Climate Experiment; GRACE, 1998) and GOCE (Gravity field and steady-state Ocean Circulation Explorer; ESA, 1999). The GOCE mission strives for a high-accuracy, high-resolution model of the Earth's static gravity field, represented by spherical harmonic coefficients complete up to

Correspondence to: R. Pail (pail@geomatics.tu-graz.ac.at) degree and order 300 , which corresponds to a shortest halfwavelength of less than $70 \mathrm{~km}$. GOCE is based on a sensor fusion concept: satellite-to-satellite tracking in the highlow mode (hl-SST) using GPS, plus on-board satellite gravity gradiometry (SGG).

The computation of the Earth's gravity model from these observations will be a laborious numerical task. This fact is not only due to the huge amount of data gathered during the two measuring periods of 6 months each, but also because of the more than 90000 spherical harmonic coefficients fully parameterizing the Earth's gravity field up to a maximum degree of 300. Some other fundamental problems arise from the spectral characteristics of the SST and SGG observation noise, or are due to the fact that the GOCE satellite is flown in a sun-synchronous orbit (inclination $i \approx 96.5^{\circ}$ ) at an average altitude of 240 to $250 \mathrm{~km}$, leaving out the polar caps where no gravity field observations are measured. This leads to a reduced numerical stability of the normal equations involved in the parameter estimation process.

The present study concentrates on the SGG component of the GOCE observables, providing information predominantly about the medium and short wavelengths of the Earth's gravity field and thus the majority of gravity field parameters. During the last decade, several approaches have been developed to solve this large system of equations (e.g. Rummel et al., 1993; Schuh, 1996; Klees et al., 2000; Gruber, 2001). In this paper we will present, compare and assess three different numerical solution techniques for the recovery of the harmonic coefficients, i.e.:

- Direct method: Parallel pcgma algorithm,

- Semi-analytic approach,

- Distributed Non-approximative Adjustment.

Although the observation equations are not identical among these methods, all processing strategies should lead to nearly identical results in terms of the 'Level 2' products (i.e. spherical harmonic coefficients, geoid heights, gravity 
anomalies). However, the individual methods show their specific characteristics, advantages, but also drawbacks, which are addressed in the present paper.

Due to the large numerical effort, advanced computational capabilities are required. In the framework of the project 'Scientific Supercomputing' at the Graz University of Technology, a parallel computing system based on the Beowulf concept is operable. At the present stage, it is composed of 24 dual-processor (866 MHz) PCs, each with 1 GB RAM and 18 GB local hard disk (Plank, 2002). The software used throughout this paper is implemented on this 'Graz Beowulf Cluster'.

However, it should be emphasized, that during the development of each parallel application the portability of the software onto different hardware architectures was taken into account. Due to the parallel quasi-standard MPI (Message Passing Interface) which was generally used, the software is not restricted to the currently used parallel platform of a Beowulf cluster, but runs, e.g. also on massive parallel systems.

\section{Functional model}

The gravitational potential $V$ of the Earth is parameterized by a harmonic series expansion in spherical coordinates $(r, \theta, \lambda)$

$$
\begin{aligned}
& V(r, \theta, \lambda)=\frac{G M}{R} \sum_{l=0}^{l_{\max }}\left(\frac{R}{r}\right)^{l+1} \\
& \sum_{m=0}^{l} \bar{P}_{l m}(\cos \theta)\left[\bar{C}_{l m} \cos m \lambda+\bar{S}_{l m} \sin m \lambda\right]
\end{aligned}
$$

where $G, M$ and $R$ are the gravitational constant, the Earth's mass and the Earth's reference radius, respectively, while $\bar{P}_{l m}$ denote the fully normalized Legendre polynomials of degree $l$ and order $m$, and $\bar{C}_{l m}, \bar{S}_{l m}$ are the corresponding fully normalized harmonic coefficients of the series expansion. The goal is now to determine the harmonic coefficients $\bar{C}_{l m}$ and $\bar{S}_{l m}$ as accurately as possible.

The non-polar satellite orbit causes data gaps at the poles, leading to a loss of orthogonality of the base functions. The resulting correlations among the base functions cause numerical instabilities, which grow dramatically with the increasing size of the polar data gap and the maximum degree of expansion (Pail et al., 2001).

Another fundamental problem is the fact that the power spectral density $S(f)$ of the gradiometer features a selected measuring bandwidth, where the observations can be measured very accurately $(S(f) \leq 4 m E / \sqrt{H z}$ within the frequency band $5 \mathrm{mHz} \leq f \leq 100 \mathrm{~m} \mathrm{~Hz}$ ), while the performance beyond this spectral window is substantially degraded (Cesare, 2002). These spectral characteristics have to be properly included in the adjustment procedure by means of filter techniques.

\section{Solution techniques}

The primary goal of all solution strategies is the retrieval of the spherical harmonic coefficients, denoted by $\bar{C}_{l m}$ and $\bar{S}_{l m}$ in Eq. (1), from the GOCE observations, i.e. the gravity gradients, in an optimum way. These gravity gradients $V_{\alpha \beta}$ are second-order spatial derivatives of the gravitational potential $V$ given in Eq. (1), where $\alpha$ and $\beta$ can take the values $x$, $y$ or $z$ representing the main spatial directions (along track, across track, radial) in an Earth-pointing coordinate frame. They compose the symmetric gravity tensor (Marussi tensor) $M=\left\{V_{\alpha \beta}\right\}$. In the case of GOCE, four components, i.e. the three main diagonal components $V_{x x}, V_{y y}$ and $V_{z z}$ as well as the off-diagonal element $V_{x z}$, are measured with very high accuracy. In the following we will briefly describe three different solution techniques for gravity field recovery from GOCE SGG observations.

\subsection{Direct method: parallel pcgma}

The main property of a direct method is that the observations are regarded as functions of the geographical location, i.e. $V_{\alpha \beta}=V_{\alpha \beta}(r, \theta, \lambda)$. In principle, the direct method is a least squares adjustment, using a standard Gauss-Markov model and applying the best linear uniformly unbiased estimation with respect to the $\Sigma^{-1}$-norm. If the gravity gradients were given as an uninterrupted time series with constant stepsize along a circular repeat orbit, the normal equation system would have a block-diagonal structure (Sneeuw, 2000). In reality, these simplifying conditions will not be strictly fulfilled, and consequently the normal equation system will deviate from the block-diagonal structure. Therefore, for the processing of real GOCE mission data, the direct method was implemented in terms of the pcgma (preconditioned conjugate gradient multiple adjustment) algorithm (Schuh, 1996). As an initialisation step, a block-diagonal preconditioner is used, and subsequently a conjugate gradient method is applied iteratively to successively improve the parameter estimates. The treatment of the gradiometer's coloured noise behaviour is performed in time domain by a discrete, linear, shift-invariant recursive filter, applied to the observation time series as well as correspondingly to the columns of the design matrix (Schuh et al., 1996; Pail and Plank, 2002).

\subsection{Semi-analytic (SA) approach}

While in the direct method the observations are regarded as functions of the geographical location $(r, \theta, \lambda)$, they can also be considered as a periodic time-series for one repeat period (Rummel et al., 1993; Sneeuw, 2000). Assuming a circular orbit, the gravitational potential $V$ in Eq. (1), and also second order derivatives $V_{\alpha \beta}$, can be rewritten as a Fourier series

$$
V_{\alpha \beta}(t)=\sum_{m} \sum_{k}\left[A_{k m} \cos \psi_{k m}(t)+B_{k m} \sin \psi_{k m}(t)\right]
$$

where $\psi_{k m}(t)$ is related to the two fundamental frequencies $\omega_{o}$ (satellite orbit revolution) and $\omega_{e}$ (Earth's rotation). 
The spherical harmonic coefficients $\bar{C}_{l m}, \bar{S}_{l m}$ of the same order $m$ are lumped together in a linear way to compose the Fourier coefficients $A_{k m}$ and $B_{k m}$ :

$$
\begin{aligned}
& A_{k m}= \\
& \frac{G M}{R} \sum_{l=l_{\min }[2]}^{l_{\max }}\left(\frac{R}{r}\right)^{l+1} \lambda_{l k}^{(\alpha \beta)} \bar{F}_{l m}^{k}(i)\left\{\begin{array}{c}
\bar{C}_{l m} \\
-\bar{S}_{l m}
\end{array}\right\}_{l-m}^{l-m} \text { odd } \\
& B_{k m}= \\
& \frac{G M}{R} \sum_{l=l_{\min }[2]}^{l_{\max }}\left(\frac{R}{r}\right)^{l+1} \lambda_{l k}^{(\alpha \beta)} \bar{F}_{l m}^{k}(i)\left\{\begin{array}{c}
\bar{S}_{l m} \\
\bar{C}_{l m}
\end{array}\right\}_{l-m}^{l-m} \text { odd }
\end{aligned}
$$

where $\bar{F}_{l m}^{k}(i)$ denotes the inclination function which depends on the orbit inclination $i$. The spectral transfer coefficients $\lambda_{l k}^{(\alpha \beta)}$ express the relation between the spherical harmonics $\bar{C}_{l m}, \bar{S}_{l m}$ and a specific observable $V_{\alpha \beta}$.

In this SA approach the Fourier coefficients ('lumped coefficients') $A_{k m}, B_{k m}$ are computed by FFT techniques in a first step, and in a second step the harmonic coefficients $\bar{C}_{l m}$, $\bar{S}_{l m}$ are adjusted order by order from the pseudo-observations $A_{k m}, B_{k m}$, assuming a strict block-diagonal structure of the normal equation system. In a strict sense, this algorithm only holds, if an uninterrupted sequence of observations with constant sampling rate is measured along a circular, exact repeat orbit. Similarly to the pcgma method, the deviations from these requirements are incorporated by means of an iterative procedure.

While in the pcgma method the gradiometer's coloured noise behaviour is treated by decorrelation applying a recursive digital filter procedure, essentially representing a convolution of the observations by a filter impulse response in time domain, in the SA approach this filter operation is a simple multiplication in frequency domain. The 'lumped coefficients' $A_{k m}, B_{k m}$ (representing the observations in the frequency domain) are directly related to a frequency via $\psi_{k m}$, and the spectral gradiometer properties can be described by the frequency-dependent variances $\sigma_{k m}^{2}$, which are included as a diagonal variance-covariance matrix of the pseudo-observations $A_{k m}, B_{k m}$ (cf. Eqs. 3 and 4) in the course of the adjustment of the harmonic coefficients. The SA approach is by far the fastest method and suggests itself as a powerful tool for quick-look validation to derive a fast diagnosis of the GOCE system performance.

\subsection{Distributed Non-approximative Adjustment (DNA)}

The basic idea of this newly proposed method is to process not only parts of the normal equation matrix, which is essentially reduced to the block-diagonal structure in the case of the direct method (pcgma) and the SA approach, but to solve the whole system in a strict sense. This requires an enormous amount of computer resources. As an example, a normal equation system complete up to degree and order 300 holds about 4.1 billion elements, and thus requires a storage of $31.1 \mathrm{~GB}$ (double precision). This huge matrix cannot be stored in the main memory of a single computer, but has to be distributed over the processing elements of the cluster.

The DNA process can be split into two parts, i.e. the setup of the normal equation matrix, and the solution of the system. Most of the computation time is used for assembling the normal equations, because for each observation the normal equation matrix has to be updated. The filtering procedure needed due to the gradiometer's coloured noise behaviour is done by applying an analogous recursive digital filter strategy as in the pcgma method (cf. Sect. 3.1).

For a distributed memory system, the data transfer between the Processing Elements (PEs) is a bottleneck. In a Beowulf cluster, this connection can slow down the whole system if huge quantities of data have to be exchanged between different nodes, leading to a side condition of minimizing the number of messages in the implementation of the DNA algorithm. Therefore, each PE was allowed to update its own part of the normal equation matrix independently from the others. Although the same calculations (e.g. setting up the observation equations) are done by more than one PE, leading (theoretically) to a loss of performance, no messages are needed during the whole assembly step.

The second part, i.e. the solution of the normal equation system, is very different concerning the overall behaviour. Since symmetric matrices are used, the solution of the system is conveniently performed by Cholesky decomposition. A second difference is the data transfer required to apply this algorithm. Each PE needs the already reduced parts from its predecessors to reduce its own part. Therefore, a close interaction between the nodes is required to solve the system. More details about the DNA algorithm can be found in Plank (2002).

Concerning the computing time, the DNA algorithm is outperformed by the two approximative solution strategies, because the number of operations dramatically increases. However, the DNA algorithm solves the system in a strict sense. Therefore, it is able to handle additional non-gravitational parameters (e.g. calibration factors, nonconservative force models) in a correct way, because it does not depend on the sparsity of the normal equation matrix. In contrast, the approximative methods rely heavily on this property, and additional correlations destroy the sparse structure of the matrix, and thus violate the initial assumption these approximative methods are based on. A second big advantage of the strict DNA solution is the fact, that it provides the full inverse and thus also the full variance-covariance matrix, which is needed by many GOCE user groups in geodesy, oceanography and geophysics.

\section{Simulation and results}

In order to assess the performance of the three numerical solution strategies for Earth's gravity field recovery regarding the quality of the recovered coefficients and the computational effort in terms of computing time, a closed-loop simulation was performed. It is based on OSU91a (Rapp et 


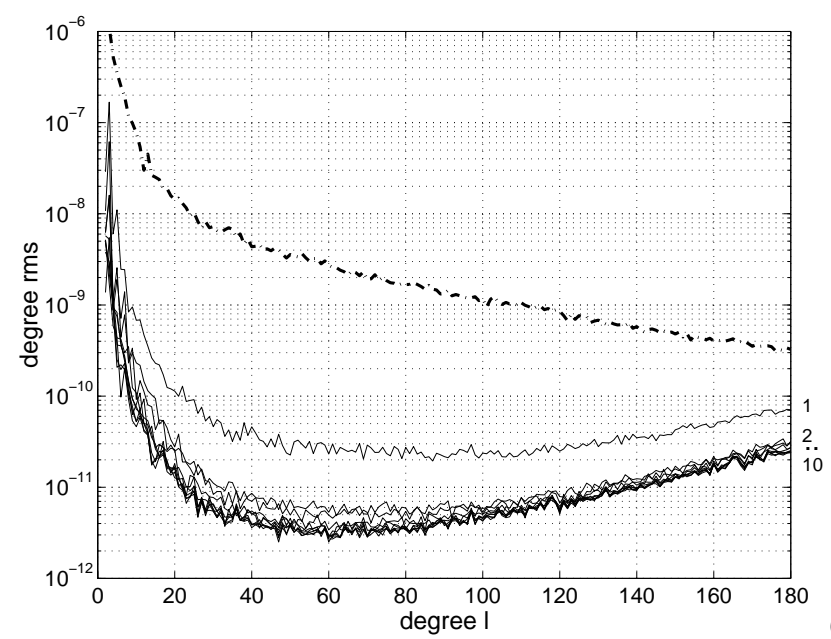

(a)

Fig. 1. Convergence behaviour of (a) the parallel pcgma and (b) the SA approach with respect to the OSU91a model (dash-dotted curve) in terms of degree rms defined in Eq. (5) for 10 iterations.

al., 1991), complete up to degree and order $l_{\max }=180$. Measurement time-series representing the gradiometer signal are computed along a non-circular, sun-synchronous 29 days/467 revolutions repeat orbit with an inclination of $i=$ $96.6^{\circ}$ and a nominal altitude of approximately $250 \mathrm{~km}$. These orbit data were generated in the framework of the 'From Eötvös to mGal' project by the Delft Institute of EarthOriented Space Research (DEOS, cf. Koop et al., 2000) applying numerical orbit integration. The simulated measurements (3 main diagonal elements of the gradient tensor: $\left.V_{x x}, V_{y y}, V_{z z}\right)$ are regularly distributed along the orbit with a sampling interval of $\Delta t=5 \mathrm{~s}$, leading to a data volume of more than 1.5 million observations (more than 500000 epochs and 3 tensor components). The test data set is restricted to a maximum degree $l_{\max }=180$, because only a reduced number of data (about one month of data with $5 \mathrm{~s}$ sampling compared to the real mission profile of two times 6 months of data with a sampling interval of $1 \mathrm{~s}$ ) is used in the present simulation.

A favourable aspect of this restriction is, that no regularization is required to solve the normal equation system, which would at least partly disguise the numerical properties of the three different methods, i.e. by using a maximum resolution of degree/order 180 the normal equation system is still regular, although due to the polar data gaps - resulting in an increased non-orthogonality of the base functions - a decreased numerical stability of the solution predominantly for the zonal and near-zonal coefficients has to be expected.

The observations were superposed by a measurement noise time-series. Since it was intended to simulate the instrument noise characteristics as realistically as possible, random variables with a specific spectral behaviour applying a general linear, causal shift-invariant stochastic process (ARMA process) according to the specifications defined in Cesare (2002) were generated.

In the following, the quality of the solutions are compared in terms of differences between the estimated coefficients and the 'true' OSU91a reference model, as well as in terms of Level 2 products such as gravity anomalies, thus closing the loop of our simulation strategy.

Figure 1 shows the convergence behaviour of the two iterative techniques in terms of the deviations of the estimated coefficients from the initial 'true' OSU91a-model represented by the degree rms

$\sigma_{l}^{(i)}=$
$\sqrt{\frac{1}{2 l+1} \sum_{m=0}^{l}\left[\left(\bar{C}_{l m, i}^{(e s t)}-\bar{C}_{l m}^{(O S U)}\right)^{2}+\left(\bar{S}_{l m, i}^{(e s t)}-\bar{S}_{l m}^{(O S U)}\right)^{2}\right]}$

for the first $i=1, \ldots 10$ iterations. Here (est) denotes the adjusted quantities and $(O S U)$ refers to our reference model OSU91a. Except for the slightly different convergence behaviour predominantly for the lower degrees $l$ (in this spectral region the SA approach shows a faster convergence), it can be stated that both methods deliver nearly identical final solutions for the geopotential coefficients.

Figure 2a shows the deviations of the harmonic coefficients estimated by the strict DNA solution from the 'true' OSU91a model. It clearly displays the well-known fact that the zonal and near-zonal coefficients can only be estimated with decreased accuracy, which is due to the sunsynchronous orbit configuration leaving data gaps at the poles (Sneeuw and van Gelderen, 1996). Since no regularization was applied in the course of the adjustment procedure, this effect is fully mapped onto the estimation results. Figure $2 \mathrm{~b}$ illustrates the gravity anomaly deviations propagated from the DNA coefficient estimates with respect to the 'true' OSU91a model.

In the Figs. $2 \mathrm{c}$ to $\mathrm{f}$, the DNA solution is used as the reference, and the deviations of the parallel pcgma algorithm (Fig. 2c and d) and the SA approach (Fig. 2e and f) from this DNA result in terms of harmonic coefficients and gravity 

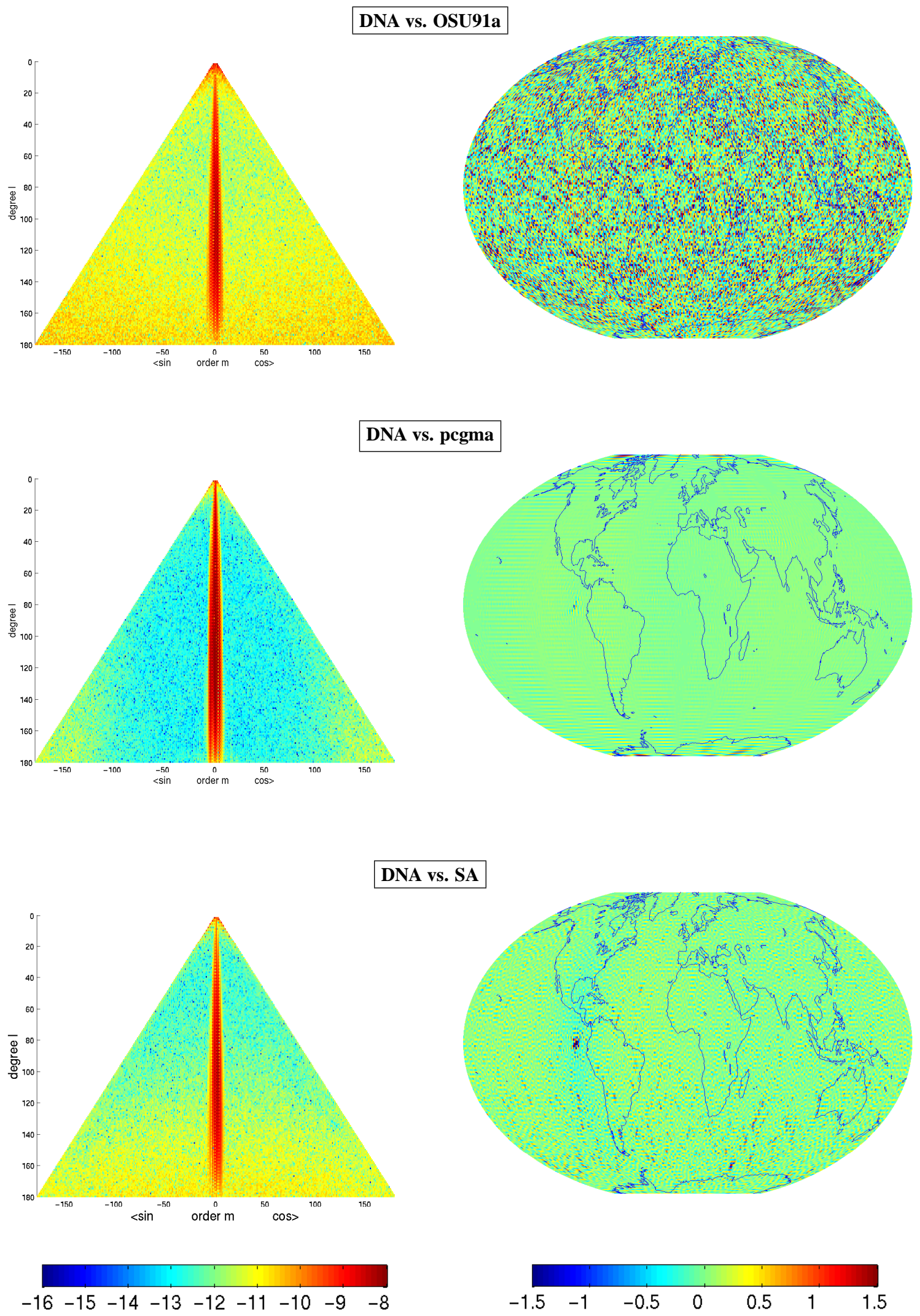

Fig. 2. Differences between the DNA approach and the "true" OSU91 a model (top), between DNA and parallel pcgma package (center), and between DNA and SA (bottom). On the left the differences between the harmonic coefficients (in $\log _{10}$-scale), and on the right in terms of gravity anomalies (in [mGal], $1 \mathrm{mGal}=10^{-5} \mathrm{~m} \mathrm{~s}^{-2}$ ) are displayed. 
Table 1. Statistical comparison of parallel pcgma, SA, and DNA methods in terms of gravity anomaly deviations $\Delta g$ [mGal] $(1 \mathrm{mGal}$ $=10^{-5} \mathrm{~m} \mathrm{~s}^{-2}$ ) from the 'true' OSU91a reference model. The last column shows the standard deviations using the DNA result as the reference. The statistics refer to 10 iterations for pcgma and 5 iterations for SA

\begin{tabular}{|l||c|c|c|c|}
\hline$(\Delta g[\mathrm{mGal}])$ & $\min ^{(O S U)}$ & $\max ^{(O S U)}$ & $\sigma^{(O S U)}$ & $\sigma^{(D N A)}$ \\
\hline \hline pcgma & -2.74 & 2.49 & 0.58 & 0.15 \\
\hline SA & -2.90 & 3.49 & 0.60 & 0.22 \\
\hline DNA & -2.44 & 2.71 & 0.56 & - \\
\hline
\end{tabular}

anomalies are plotted. Obviously, the deviations of the solutions from the 'true' OSU91a reference model, which reflect the coloured noise superimposed onto the SGG observations, are considerably larger than the differences among the individual adjustment solutions. This is underpinned by Table 1 , which summarizes the main statistical parameters for these gravity anomaly difference fields.

The pcgma method shows slightly lower deviations from the DNA than the SA approach, which can be explained by the fact, that the former two methods use identical filter strategies in time domain to cope with the gradiometer's coloured noise characteristics, whereas the SA method applies a filter in frequency domain. Concerning the storage requirements and the run-time behaviour, there are considerable differences between the three methods, as it is summarized in Table 2.

\section{Discussion and conclusions}

From a theoretical point of view and neglecting numerical aspects, the DNA solution of the full normal equation system and iterative methods such as the pcgma algorithm using a sparse matrix approximate should lead to identical results, provided that the requirements of convergence are fulfilled, and the iterative process is truncated properly. In principle, this holds also for the iterative SA method. However, since a different approach to model colored noise - which is fast, but approximative - is applied, deviations from the DNA results have to be expected, but they turn out to be small compared with other effects influencing the solution. Correspondingly, the closed-loop simulation performed in section 4 demonstrates that the discrepancies among the three solution strategies are far below the noise level.

Concerning the computation time, the SA approach is definitely the fastest, which is due to the fact that it works partially in the frequency domain. Therefore, the SA approach can be used as a tool for quick-look validation to derive a fast diagnosis of the GOCE system performance. Considering the computing time, the two approximative and thus iterative methods (parallel pcgma and SA) by far outperform the strict DNA approach, which is due to the different number of elements of the normal equations taken into account
Table 2. Storage requirements for the normal equation matrices and performance parameters (CPU time) needed by the three methods for a full parameterization up to degree $l_{\max }=180$

\begin{tabular}{|l||c|c||c|c|c|}
\hline method & $\begin{array}{c}\text { no. elements } \\
\text { (millions) }\end{array}$ & $\begin{array}{c}\text { storage requ. } \\
\text { (MB) }\end{array}$ & no. PEs & no. iter. & $\begin{array}{c}\text { total CPU } \\
\text { time }\end{array}$ \\
\hline \hline pcgma & 1 & 8 & 25 & 10 & $12^{h} 20^{\text {min }}$ \\
\hline SA & 1 & 8 & 1 & 5 & $20^{\text {min }}$ \\
\hline DNA & 537 & 4300 & 49 & - & $348^{h}$ \\
\hline
\end{tabular}

in the processing (cf. Table 2), leading to a giant number of additional operations that have to be performed by the DNA.

On the other hand, the advantage of the DNA approach is that it solves the whole normal equation system in a strict sense. In the processing of the real mission, additional nongravitational parameters (e.g. calibration parameters, nonconservative forces, etc.), and maybe also parameters describing the temporal variations of the Earth's gravity field, will have to be introduced into the parameter vector. In this case, strong correlations between these additional components and the spherical harmonic coefficients will occur, destroying the dominant block-diagonal structure of the normal equations. Among the three methods the DNA approach is best able to handle these additional parameters in a correct way, because it does not depend on the sparsity of the normal equation matrix. Additionally, it provides the full inverse and thus the full variance-covariance information of the estimated gravity field parameters.

Acknowledgements. This study was partly performed in the course of the GOCE project "From Eötvös to mGal+", funded by the European Space Agency (ESA) under contract No. 14287/00/NL/DC.

\section{References}

Cesare: Performance requirements and budgets for the gradiometric mission, Technical Note, GOC-TN-AI-0027, Alenia Spazio, Turin, Italy, 2002.

ESA: Gravity Field and Steady-State Ocean Circulation Mission. Reports for mission selection, The four candidate Earth explorer core missions, SP-1233(1), European Space Agency, Noordwijk, 1999.

ESA: GOCE End-to-End Closed Loop Simulation, Final Report, European Space Agency, Noordwijk, 2000.

GRACE: Gravity Recovery and Climate Experiment: Science and Mission Requirements Document, revision A, JPLD-15928, NASA's Earth System Science Pathfinder Program, Jet Propulsion Laboratory, Pasadena, CA., 1998.

Gruber, T.: High-resolution gravity field modeling with full variance-covariance matrices, J. Geod., 75, 505-514, 2001.

Klees, R., Koop, R., Visser, P. N. A. M., and van den IJssel, J.: Efficient gravity field recovery from GOCE gravity gradient observations, J. Geod., 74, 561-571, 2000.

Koop, R., Visser, P. N. A. M., van den IJssel, J., and Klees, R.: Detailed scientific Data Processing Approach. ESA project "From 
Eötvös to mGal" (Ed) Sünkel, Final report, ESA/ESTEC Contract 13392/98/NL/GD, European Space Agency, Noordwijk, 29-103, 2000.

Pail, R., Plank, G., and Schuh, W.-D.: Spatially restricted data distribution on the sphere: the method of orthonormalized functions and applications, J. Geod., 75, 44-56, 2001.

Pail, R. and Plank, G.: Assessment of three numerical solution strategies for gravity field recovery from GOCE satellite gravity gradiometry implemented on a parallel platform, J. Geod., 76, 462-474, 2002.

Plank, G.: Implementation of the pcgma-package on massive parallel systems. ESA project, "From Eötvös to $\mathrm{mGal}+$, Final report, WP 3, ESA/ESTEC Contract 14287/00/NL/DC, European Space Agency, 183-216. 2002.

Rapp, R., Wang, Y., and Pavlis, N.: The Ohio state 1991 geopotential and sea surface topography harmonic coefficient models, OSU Report 410, Department of Geodetic Science and Surveying, The Ohio State University, Columbus, 1991.

Reigber, Ch., Schwintzer, P., and Lühr, H.: CHAMP geopotential mission, Boll. Geof. Teor. Appl., 40, 285-289. 1999.

Rummel, R., van Gelderen, M., Koop, R., Schrama, E., Sansó,
F., Brovelli, M., Miggliaccio, F., and Sacerdote, F.: Spherical harmonic analysis of satellite gradiometry, Neth. Geod. Comm., Publications on Geodesy, no. 39, Delft, The Netherlands, 1993.

Schuh, W.-D.: Taylored Numerical Solution Strategies for the Global Determination of the Earth's Gravity Field, Mitteilungen geod. Inst. TU Graz, no. 81, Graz Univ. of Technology, Graz, 1996.

Schuh, W.-D., Sünkel, H., Hausleitner, W., and Höck, E.: Refinement of Iterative Procedures for the Reduction of Spaceborne Gravimetry Data, ESA project CIGAR IV, Final report. ESA contract 152163-ESA study ESTEC/JP/95-4-137/MS/nr, European Space Agency, Noordwijk, 157-212, 1996.

Sneeuw, N.: A semi-analytical approach to gravity field analysis from satellite observations, Dissertation, DGK, Reihe C, Munich, no. 527, Bayerische Akademie d. Wissenschaften, Munich, 2000.

Sneeuw, N. and van Gelderen, M.: The polar gap, in: Geodetic boundary value problems in view of the one centimeter geoid, (Eds) Sanso, F. and Rummel, R., Lecture Notes in Earth Sciences, 65, Springer, 559-568, 1997. 